\title{
Ultrafine Structure of Calcific Deposits Developed in Calcific Tendinopathy
}

\author{
Marketa Zelenková1, Otakar Söhnel'2, Felix Grases ${ }^{3 *}$ \\ ${ }^{1}$ TOSEDA s. r. o., Pardubice, Czech Republic \\ ${ }^{2}$ Faculty of Environmental Studies, University of J.E. Purkyně, Ústí n.L., Czech Republic \\ ${ }^{3}$ Laboratory of Renal Lithiasis Research, University Institute of Health Sciences Research (IUNICS), University of \\ Balearic Islands, Palma of Mallorca, Spain \\ Email: ${ }^{*}$ fgrases@uib.es
}

Received 26 February 2015; accepted 27 March 2015; published 31 March 2015

Copyright (C) 2015 by authors and Scientific Research Publishing Inc.

This work is licensed under the Creative Commons Attribution International License (CC BY). http://creativecommons.org/licenses/by/4.0/

(c) () Open Access

\begin{abstract}
The ultrafine structure of tendons deposits formed in three patients, males aged 52 and 61 years and a female aged 71 years were evaluated by atomic force microscopy. Three distinctly different structures of deposit surface were identified: (i) compact, smooth and uneven surface composed of closely packed nanoparticles of diameter $30 \mathrm{~nm}$; (ii) surfaces consisting of plate-like crystalline particles about $30 \mathrm{~nm}$ thick that formed larger entities divided by deep depressions; (iii) rough surface formed by individual or closely attached elongated needle-like particles with elliptical cross-section of diameter about $30 \mathrm{~nm}$. These surface structures were developed by different formation mechanisms: (i) Aggregation of Posner's clusters into nanoparticles formed on biological calcific able surfaces and in the bulk of body fluid surrounding the deposits that subsequently settled onto the deposit surface; (ii) Regular crystal growth on surface nuclei generated at low supersaturation of body fluid with respect to the phosphatic phase and/or in a narrow cavity containing a very limited volume of liquid; (iii) Solution mediated re-crystallization of the upper layers of a deposit or unstable crystalline growth governed by volume diffusion of building units to the particle tip. Small rods, $40 \mathrm{~nm}$ wide and from 100 to $300 \mathrm{~nm}$ long, with no apparent order were detected only on the surface of deposit formed in the female patient. These rods could be debris of collagen fibres that disintegrated into individual building units (macromolecules) with some showing breakdown into smaller fragments.
\end{abstract}

\section{Keywords}

Calcific Tendinopathy, Carbonated Hydroxyapatite, Posner's Clusters, Deposit Structure, Formation Mechanism

\footnotetext{
"Corresponding author.
} 


\section{Introduction}

Calcification of the rotator cuff tendons is a common alteration affecting adults. In general, these calcifications tend to be reabsorbed after a period of acute pain. However, the pathogenesis of calcific tendinopathy is not yet fully understood. It is assumed that under favourable conditions a process of cell-mediated calcification proceeds resulting in the concretion formation [1].

The composition and structure of tendons deposits and factors influencing their formation were recently investigated in concretions from 29 patients of age 31 to 78 years [2]. Deposits consisted of organic matter and poorly crystalline carbonated hydroxyapatite. The phosphatic phase appeared as ellipsoidal objects ranging in size from 20 to $200 \mu \mathrm{m}$ and small spheres $5 \mu \mathrm{m}$ in diameter, with both types of particles having microscopically smooth surfaces.

To clarify the mechanism of particle formation, this study utilized atomic force microscopy (AFM) to investigate the ultrafine structure of these particles.

\section{Experimental}

The studied deposits (No. 13, 15 and 16) had been removed from three patients, two males aged 52 and 61 years and a female aged 71 years analyzed previously [2]. Deposits were removed using an ultrasound guided needle followed by repeated injections of saline solution and aspiration of the suspended solids. Solids were filtered out and dried. Fragments of deposits were very fragile and readily disintegrated into a fine powder. The deposits were dispersed in $96 \%$ ethanol using sonification; several drops of formed suspension were placed on a glass holder and dried. All samples consisted of individual spherical particles with sizes ranging from approximately 20 to $40 \mu \mathrm{m}$, larger spherical particles of $70 \mu \mathrm{m}$ in diameter and occasional aggregates up to $200 \mu \mathrm{m}$ in size. Each sample was initially observed with an optical microscope accompanying the AFM. The individual particles and sites on surface of each sample were randomly selected for detailed study by AFM.

Surface of particles was observed by Atomic Force Microscope Dimension Icon from Bruker USA (Bruker, Santa Barbara, CA) in the Peak Force QNM Imagining Mode, This method provided nanoscale topographic, elastic modulus and adhesion maps of the particle surface.

The red and yellow regions in the AFM topographic images corresponded to depressions below and projections above the surface. The darker red indicated a deeper depression, whereas a lighter yellow indicated a greater protrusion. On nanoscale elastic modulus maps showing variations of the surface modulus (the Derjaguin-Müller-Toporov modulus), the red and yellow colors indicated sites composed of soft and stiff matter, respectively. On nanoscale adhesion maps showing adhesion of the probe tip to the surface, the red and yellow colours indicated sites composed of soft and stiff matter, respectively.

\section{Results}

Figure 1 shows the appearance of disintegrated tendons deposits; consisting of organic matter, small spherical particles and large agglomerates. The surfaces of particles and agglomerates from the three deposits exhibited various nanoscale characteristics:

1. Compact, smooth, slightly uneven surface with occurrence of protrusions and depressions of various heights and depths composed of closely arranged spherical particles 25 to $40 \mathrm{~nm}$ in size, Figure 2(a), or similarly compact, but rough and markedly uneven surface formed by spherical particles of diameter about $30 \mathrm{~nm}$ from which well-defined crystals protruded, Figure 2(b). A map of adhesion (not shown here) indicated that the surfaces were composed of stiff material. A 2-D image of the same site showed that the nanoparticles formed irregular spherical objects, about $0.5 \mu \mathrm{m}$ in diameter, divided by depressions 20 to $50 \mathrm{~nm}$ deep, Figure 2(a).

2. Some sites were composed of plate-like particles roughly $30 \mathrm{~nm}$ thick of crystalline appearance oriented perpendicular to the surface. These plate-like particles were either attached by lateral faces forming larger blocks divided by deep depressions or were tightly packed in no apparent order, Figure 3.

3. Elongated needle-like particles with elliptical cross-section about $30 \mathrm{~nm}$ in diameter were observed perpendicular to the surface forming rough surfaces, Figure 4. These particles were either closely arranged or attached to one another forming compact, but uneven surfaces or were individual, arranged in no apparent order and divided by deep depressions resulting in non-compact surfaces. 


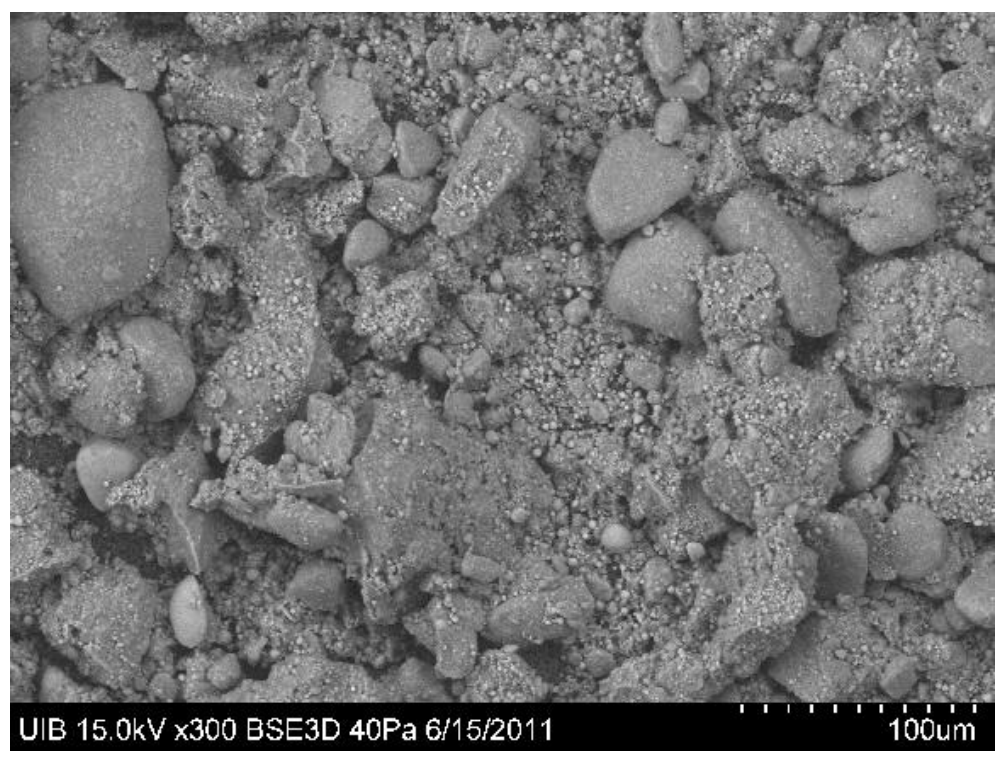

Figure 1. Electron scanning microscopic image of a disintegrated tendon deposit.

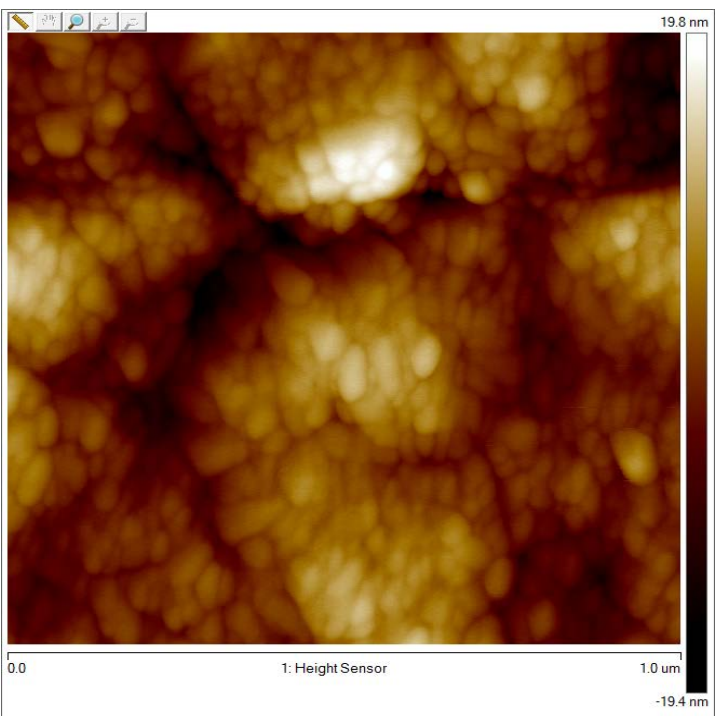

(a)

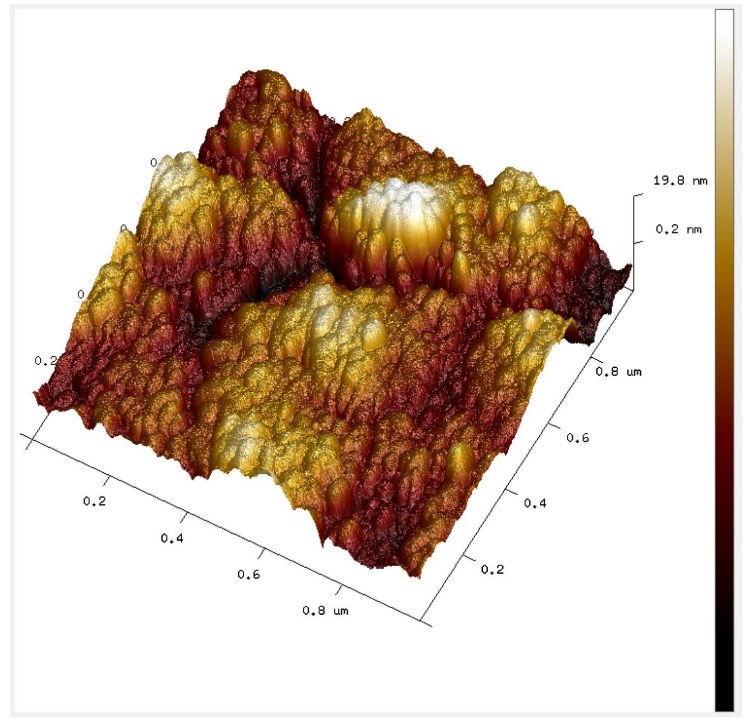

(b)

Figure 2. (a) 2-D topographic map of a compact and smooth surface of deposit No. 13. Scanned area $1 \times 1 \mu \mathrm{m}$; (b) 3-D topographic map of compact and rough surface of deposit No. 13. Scanned area $1 \times 1 \mu \mathrm{m}$.

4. Several sites on the surface of the deposit No. 16 contained small rods $40 \mathrm{~nm}$ wide and varying in length from 100 to $300 \mathrm{~nm}$ arranged without any apparent order, Figure 5(a), Figure 5(b). The structure of the underlying uneven surfaces was identical to those described in (i). Similar sites were not detected on the surfaces of deposits No. 13 and 15. Rods exhibited medium stiffness as evident from their prevailing light brown colour, Figure 5(b).

The structural characteristics described in (i), (ii) and (iii) were observed on all 3 deposits, whereas the structural features described in (iv) were observed only on the surface of particles of deposit No. 16.

\section{Discussion}

Tendons deposits i.e. deposits No. 13, 15 and 16, were composed of poorly crystalline hydroxyapatite and an 


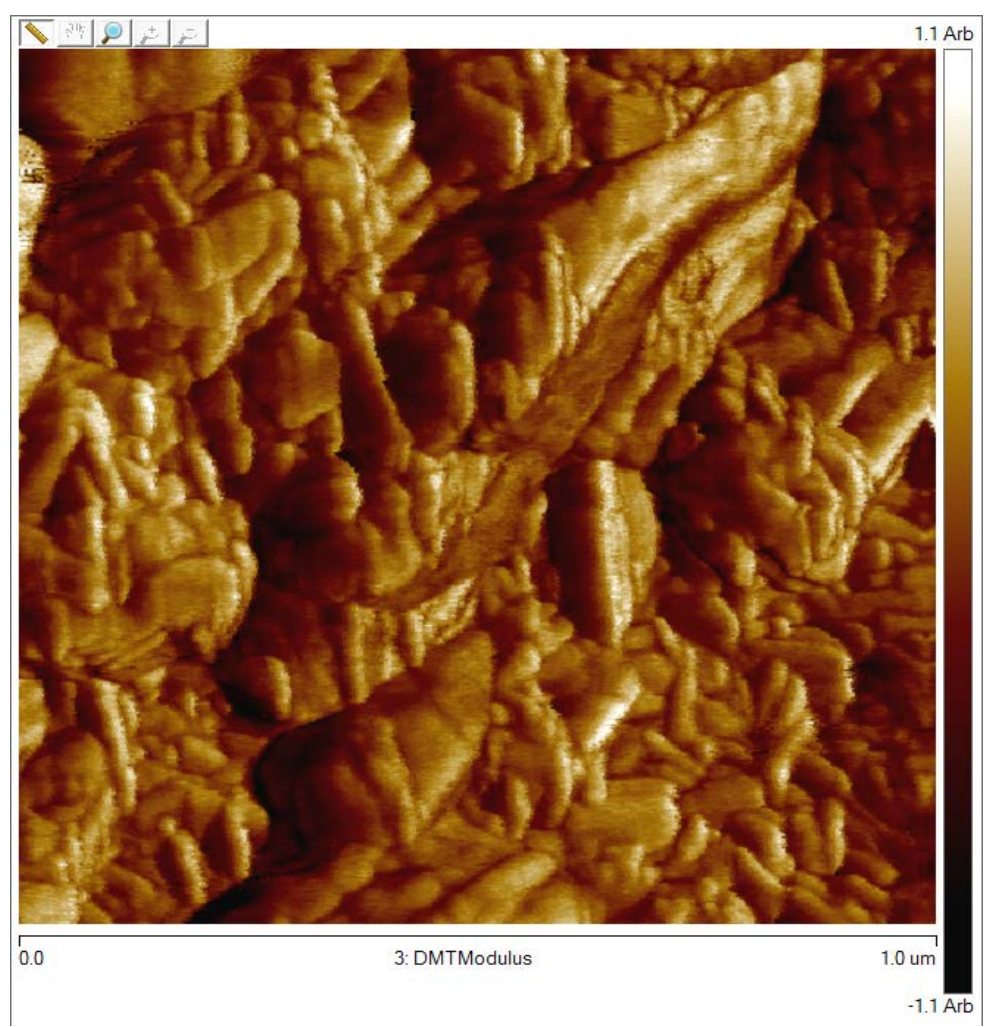

Figure 3. 3-D elastic modulus map of the surface of deposit No. 13 composed of plate-like particles. Scanned area $1 \times 1 \mu \mathrm{m}$.

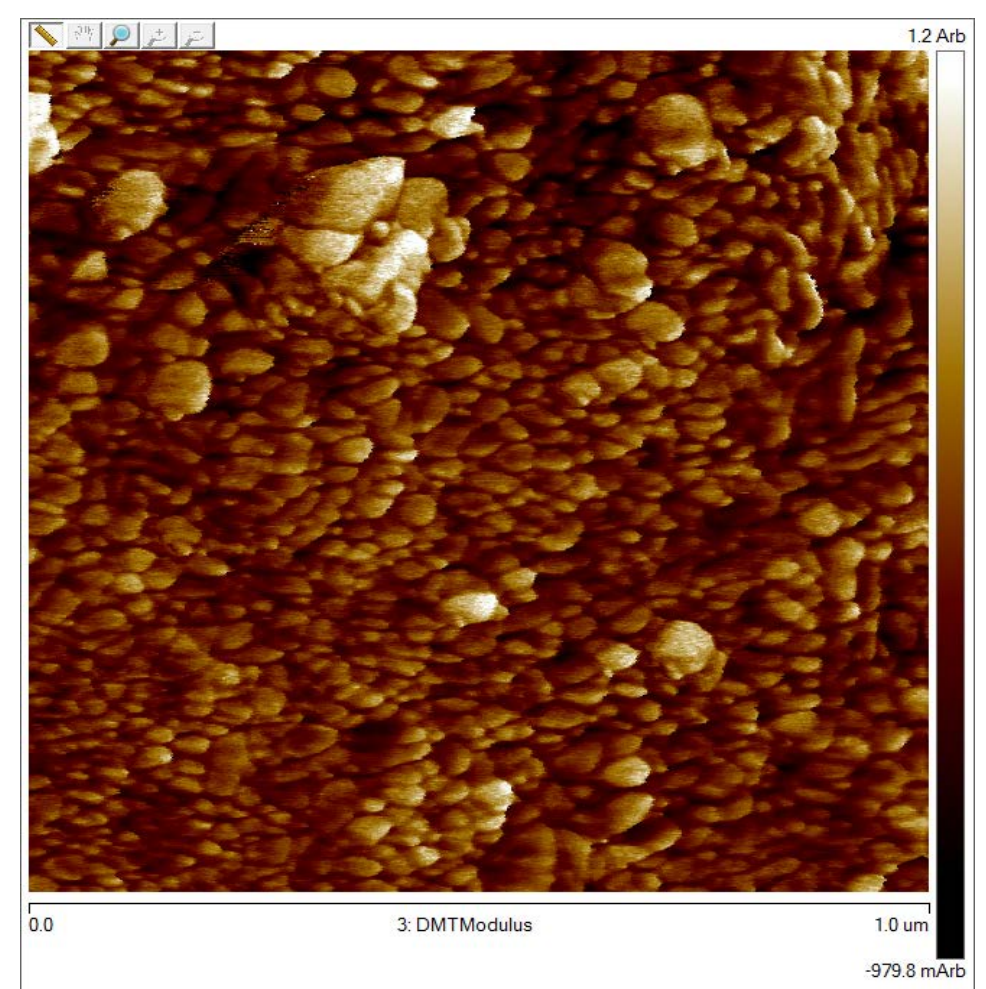

Figure 4. 3-D elastic modulus map of surface of deposit No. 15 composed of elongated needle-like particles. Scanned area $1 \times 1 \mu \mathrm{m}$. 


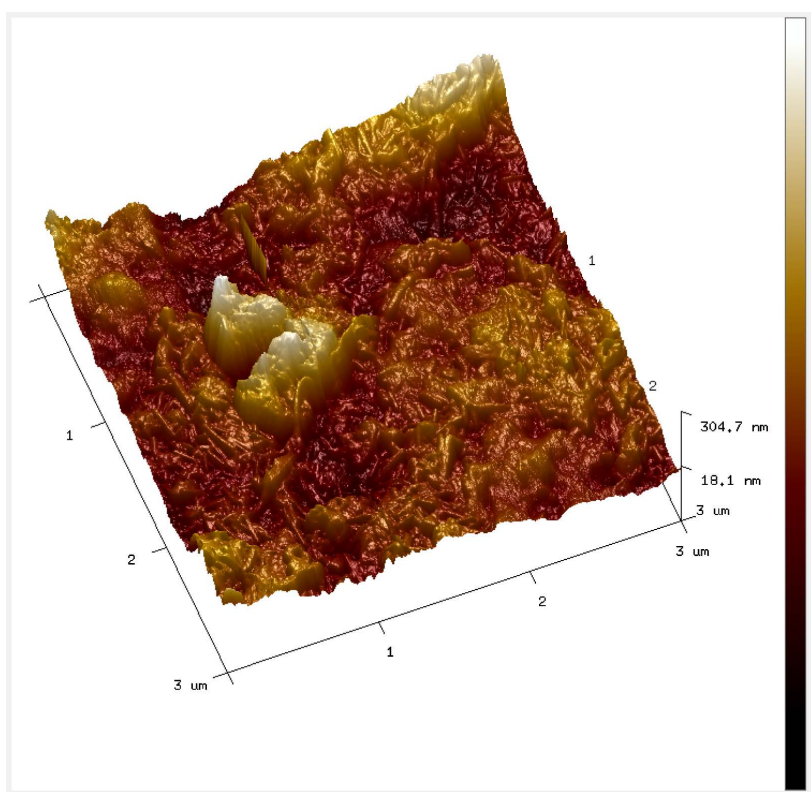

(a)

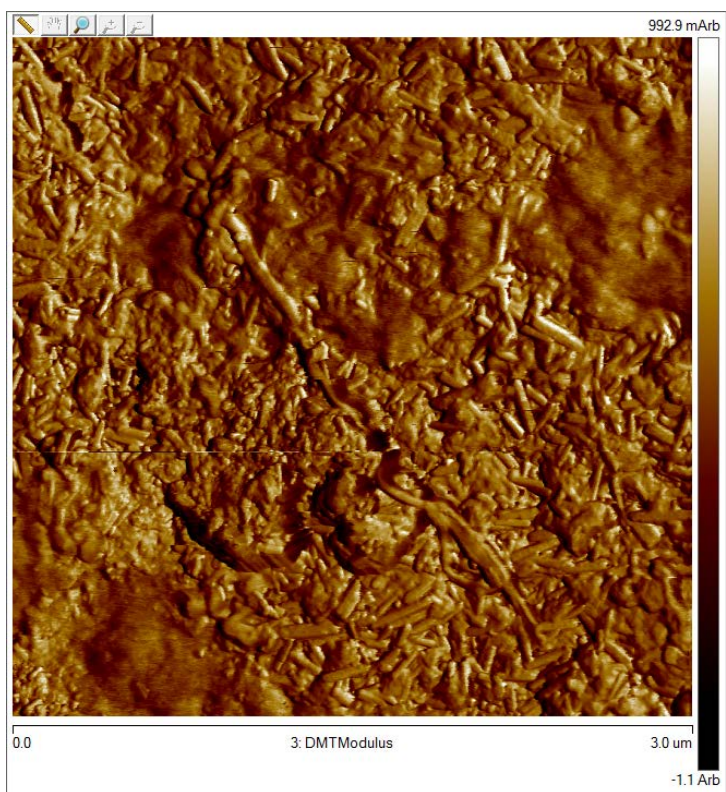

(b)

Figure 5. (a) 3-D topographic map of the surface of deposit No. 16 containing small rods. Scanned area $3 \times 3 \mu \mathrm{m}$; (b) 3-D elastic modulus map of the same site of deposit No. 16. Scanned area $3 \times 3 \mu \mathrm{m}$.

organic matter, as found in the previous study [2]. Method used at deposit removal, namely disintegration and filtration of suspension, changed completely initial spatial structure of deposit and hence only structure of individual particles could be studied.

The surfaces of all solid particles both of small spheres and larger spherical objects, were always uneven differing in height by tens of nanometres. Structural characteristic (i) to (iii) described above on the surfaces of both small spheres and large aggregates were detected on all three deposits suggesting that the mechanism involved in the formation of these deposits were similar. Moreover the finding of three distinct structural characteristics were on the surfaces of these deposits suggests that three mechanisms were involved in the formation of these tendon deposits.

Spherical nanoparticles around $50 \mathrm{~nm}$ in diameter form spontaneously by aggregation of Posner's clusters in body fluid supersaturated with respect to phosphatic phase [3] [4]. Aggregation proceeds by perikinetic coagulation in the bulk liquid and is promoted by higher supersaturation of body fluid [3] [5]. As supersaturation increases, so does the concentration of Posner's clusters, increasing the rate of coagulation resulting in the formation of larger agglomerates within the same period of time [6]. Aggregation is also promoted by the presence of biological calcific able surfaces [3]. Aggregates of the phosphatic phase formed in bulk liquid subsequently settle onto biological surfaces, where calcification proceeds by surface nucleation, and are incorporated into deposits. Thus deposits with compact and uneven surfaces are composed of closely packed nanosized particles. These structural characteristics suggest that the described mechanism is active in tendon deposit formation and may be responsible for the structures described in (i) above. Similar structure, i.e. uneven surfaces almost exclusively composed of dense spherical particles 10 to $15 \mathrm{~nm}$ in diameter were observed in some parts of fin bones of zebrafish [7].

The surface structure described in (ii) above was formed by regular crystal growth on surface nuclei originated on biological template [8] since phosphatic phase formed in the absence of proper organic template formed spatial objects consisting of irregularly intergrown thin flakes [9]. Regular plate-like crystals, which are often stuck together in larger entities separated by depressions of various depths, could develop under specific conditions, namely either at low supersaturation of body fluid with respect to the phosphatic phase and/or in narrow cavities containing very limited volumes of liquid. The rate of aggregation is depressed at low supersaturation making the size of formed agglomerates too small to settle. Similarly, agglomerates too small to settle develop in an insufficient volume of liquid that cannot contain, even at higher supersaturation, a sufficient quantity of Posner's clusters for formation of large enough entities. Whether one or both of these conditions, in addi- 
tion to the presence of organic template, predominates during the development of structure (ii) cannot be determined at present.

Rough surfaces composed of closely and/or loosely arranged needle-like particles perpendicular to the deposit surface were described in (iii) above. Individual particles were not branched and seemed to be crystals rather than objects composed of spherical aggregates stuck on top of one another. Loosely arranged needle-like particles resemble metal whiskers rather then dendrites, but their formation mechanism is still unknown. These structures may result from solution mediated re-crystallization of outer deposit layers in prolonged contact with body fluid or from unstable crystalline growth governed by volume diffusion of building units to the particle tip [10].

Structural features appearing on the surfaces of tendon deposits, specifically features (i) and (iii), are very similar to structures identified in calcific deposits located in the interior of human aortic valves [5]. Thus, the mechanism of formation of both deposit types may be similar to some extent. Deposits in both tendons and aortic valves form in stagnant liquid of comparable composition with both being supersaturated with respect to the phosphatic phase. Difference between conditions prevalent during their development is the size of volume of liquid surrounding deposit. The aortic valve deposit develops in a very limited volume of liquid, in fact its surface is just wetted by a thin layer of fluid. Tendons deposit predominantly develops in a relatively spacious cavity filled with body fluid, volume of which is sufficient for formation of large enough agglomerates that can settle onto surface of deposit ensued by incorporation into its body. However, situation when tendons deposit develops, even for a certain time, in a very limited volume of liquid is also rather realistic, as signifies the case (ii) in the previous section.

Aortic valve deposits developing in an inadequate volume of liquid are composed of crystalline matter with no evidence of nanosized spherical objects. In contrast, tendon deposits developing in sufficient volume of liquid consist predominantly of closely arranged nanosized particles. The presence of plate-like crystalline particles on tendon deposits indicates that different conditions predominated during their formation. These conditions are similar to those involved in the formation of aortic valve deposits.

The presence of large numbers of rods on the deposit surface was only observed for deposit No. 16 obtained from the oldest patient (age 71 years). The shape, size and appearance of these rods strikingly resemble the building units derived from collagen fibres, consisting of $40 \mathrm{~nm}$ wide and $300 \mathrm{~nm}$ long [11]. These rods could derive from collagen fibres that disintegrated into individual building units (macromolecules) with some showing further breakdown into smaller fragments because of physical activity. The intensity of this destructive process increases with patient age perhaps explaining why similar rods were not observed on the surface of deposits obtained from younger patients (deposits No. 13 and 15).

The arrangement of rods on the surface signified their arrival on the surface by settling from the liquid phase. Rod surfaces, however, was not soft as would correspond to an organic matter, but medium stiff. This fact suggests that their surfaces were, probably already in the liquid phase before settling, covered by a layer of amorphous phosphatic phase, an elastic modulus, i.e. stiffness, of which attains only medium values between that of organic matter and crystalline phase. Calcification may have been induced by carboxylate groups originating on the surface of macromolecules as a result of oxidation [12].

\section{Conclusion}

AFM examination of the surface structural characteristics of the phosphatic phase of tendon deposits indicating three mechanisms was involved in their formation: agglomeration of Posner's clusters into nanosized spherical objects, regular crystal growth on surface nuclei, and solution mediated re-crystallization of deposit outer layers. The involvement of each mechanism depended on the volume of body fluid surrounding the deposit and/or the actual supersaturation of body fluid with respect to the phosphatic phase.

\section{Conflicts of Interests}

The authors declare that they have no conflicts of interests.

\section{Acknowledgements}

This study was supported by a grant from the University of the Balearic Islands and by project CTQ201018271/PPQ from the Ministerio de Ciencia e Innovación (Gobierno de España), FEDER funds (European Union) 
and the project grant 9/2011 from the Conselleria d’Educació, Cultura i Universitat (Govern de les Illes Balears).

\section{References}

[1] Uthoff, H.K. and Loehr, J.W. (1997) Calcific Tendinopathy of the Rotator Cuff: Pathogenesis, Diagnosis and Management. Journal of the American Academy of Orthopaedic Surgeons, 5, $183-91$.

[2] Grases, F., et al. (2015) Morphological Characterization of Calcific Deposits Developed in Calcific Tendinopathy. Role of Crystallization Inhibitors and Regulants. Journal of Orthopaedic Research, in press.

[3] Dey, A., Bomans, P.H.H., Muller, F.A., Will, J., Frederik, P.M., de With, G. and Sommerdijk, N.A.J.M. (2010) The Role of Prenucleation Clusters in Surface-Induced Calcium Phosphate Crystallization. Nature Materials, 9, $1010-1014$. http://dx.doi.org/10.1038/nmat2900

[4] Peng, H.H., Wu, Ch.Y., Young, D., Martel, J., Young, A., Ojcius, D.M., Lee, Y.-H. and Young, J.D. (2013) Physicochemical and Biological Properties of Biomimetic Mineralo-Protein Nanoparticles Formed Spontaneously in Biological Fluid. Small, 9, 2297-2307. http://dx.doi.org/10.1002/smll.201202270

[5] Grases, F., Söhnel, O. and Zelenková, M. (2014) Ultrafine Structure of Human Aortic Valve Calcific Deposits. Journal of Cytology \& Histology, 5, 214.

[6] Ziff, R.M., McGrady, E.D. and Makin, P. (1985) On the Validity of Smoluchowski’s Equation for Cluster-Cluster Aggregation Kinetics. The Journal of Chemical Physics, 82, 5269-5274. http://dx.doi.org/10.1063/1.448600

[7] Mahamid, J., Sharir, A., Addadi, L. and Weiner, S. (2008) Amorphous Calcium Phosphate Is a Major Component of the Forming Fin Bones of Zebrafish: Indications for an Amorphous Precursor Phase. Proceedings of the National Academy of Sciences, 105, 12748-12753. http://dx.doi.org/10.1073/pnas.0803354105

[8] Colfen, H. (2010) A Crystal-Clear View. Naturals Materials, 9, 960-961. http://dx.doi.org/10.1038/nmat2911

[9] Wang, L. and Nancollas, G.H. (2009) Pathways to Biomineralization and Biodemineralization of Calcium Phosphates: The Thermodynamics and Kinetic Control. Dalton Transactions, 2665-2672. http://dx.doi.org/10.1039/b815887h

[10] Glicksman, M.E. and Lupulescu, A.O. (2004) Dendritic Crystal Growth in Pure Materials. Journal of Crystal Growth, 264, 541-549. http://dx.doi.org/10.1016/j.jcrysgro.2003.12.034

[11] Ottani, V., Raspanti, M. and Ruggeri, A. (2001) Collagen Structure and Functional Implications. Micron, 32, 251-260. http://dx.doi.org/10.1016/S0968-4328(00)00042-1

[12] Takeuchi, A., Ohtsuki, Ch., Miyazaki, T., Kamitakahara, M., Ogata, S., Yamazaki, M., Furutani, Y., Kinoshita, H. and Tanihara, M. (2005) Heterogeneous Nucleation of Hydroxyapatite on Protein: Structural Effect of Silk Sericin. Journal of The Royal Society Interface, 2, 373-378. 